\title{
Die Dorfapp als Ersatz für die Dorfkneipe?
}

\section{Erfahrungen aus einem Dorf in der Vulkaneifel}

\author{
Ariane Sept ${ }^{1}$ \\ Eingegangen: 18. April 2020 / Überarbeitet: 6. Oktober 2020 / Angenommen: 7. Oktober 2020 / Online publiziert: 30. Oktober 2020 \\ (c) Der/die Autor(en) 2020
}

\section{Zusammenfassung}

Immer mehr Dörfer nutzen sogenannte Dorfapps. Teilweise sollen diese verlorenen gegangene Treffpunkte und Kommunikationsorte wie Läden oder Kneipen ersetzen. Aber kann eine Dorfapp die Dorfkneipe ersetzen? Dazu werden im Artikel am Beispiel des Dorfes Dreis-Brück in Rheinland-Pfalz und seiner Dorfapp die unterschiedlichen Funktionen der Kneipe systematisiert und mit den Möglichkeiten der Dorfapp verglichen. Im Zentrum steht die Dorfkneipe als Informationsund Kommunikationsort, aber auch ihre Rolle als Treffpunkt, Ort der Entspannung, Ankunftsort sowie als Emotions- und Erinnerungsraum wird beleuchtet. Soll eine Dorfapp teilweise Funktionen von verloren gegangenen Kneipen übernehmen, kann eine systematische Betrachtung der Probleme, die mithilfe digitaler Tools gelöst werden sollen, im Kontext einer Systematisierung von analogen Raumeigenschaften hilfreich sein, um letztlich Verknüpfungen zwischen digitalen Angeboten und analogen Orten zu entwickeln. Der Artikel versteht sich als Beitrag zur raumbezogenen Auseinandersetzung mit gastronomischen Einrichtungen und zur Rolle von Digitalisierung in ländlichen Räumen.

Schlüsselwörter Digitalisierung · Gastronomie $\cdot$ Ländlicher Raum · Dorfentwicklung $\cdot$ Kommunikation

\section{The village app as a substitution for the village pub?}

Experience of a village in Vulkaneifel, Germany

\begin{abstract}
More and more villages have started using village apps. In some cases, these are intended to replace lost meeting places and places of communication such as shops or pubs. But can a village app replace the village pub? In the article, the example of the village of Dreis-Brück in Rhineland-Palatinate and its village app is used to systematize the different functions of the pub and compare them with those of the app. The focus is on the village pub as a place of information and communication, but its role as meeting point, place of relaxation, arrival point, as well as a space for emotions and memories, is also examined. If a village app can partially take over functions of lost village pubs, a systematic consideration of the problems to be solved with the help of digital tools can be helpful in the context of a systematization of the analog spatial entities, to ultimately develop links between digital and analog places. The article is intended as a contribution to the spatial analysis of gastronomic facilities and the role of digitalization in rural areas.
\end{abstract}

Keywords Digitalization $\cdot$ Gastronomy $\cdot$ Rural areas $\cdot$ Village development $\cdot$ Communication

Dr. Ariane Sept

ariane.sept@leibniz-irs.de

1 Leibniz-Institut für Raumbezogene Sozialforschung (IRS), Flakenstr. 29-31, 15537 Erkner, Deutschland
Die Auseinandersetzung mit Gaststätten wurde in der raumbezogenen Wissenschaft und Praxis Deutschlands jahrelang vernachlässigt, obwohl städtische Gaststätten „für die Aufenthaltsqualität, das Image und die Identifikation mit einem Standort von erheblicher Bedeutung [sind]" (Franz 2020, S. 97). In den wenigen raumbezogenen Arbeiten zu ga- 
stronomischen Einrichtungen (z.B. Pätzold 2012; Dröge und Krämer-Badoni 1987; Gyr 1997; Franz 2019, 2020) steht die städtische Gaststätte im Fokus. Daneben lässt sich im medialen Diskurs eine wiederkehrende Berichterstattung über das Sterben der Dorfkneipe beobachten (z. B. Bade und Nehls 2018; Norddeutscher Rundfunk 2020). Wissenschaftliche Veröffentlichungen verweisen zwar auf den Wegfall von Gaststätten in Dörfern (z. B. Franz 2020; Henkel 2018; Sixtus et al. 2019), dies wird jedoch - von wenigen Ausnahmen abgesehen (z.B. Hopfinger et al. 2013) - eher als problematische Tatsache berichtet und weniger in seiner Bedeutung beleuchtet.

In einem laufenden Forschungsprojekt zu sozialen Innovationen und Digitalisierungen in ländlichen Räumen ist das Dorf Dreis-Brück im Landkreis Vulkaneifel in Rheinland-Pfalz eine von mehreren Fallstudien, da es durch die Nutzung einer Dorfapp auffiel. Die im September 2019 und Februar 2020 geführten zwölf qualitativen, semistrukturierten Interviews mit zwei Verwaltungsangestellten sowie 16 Bewohnerinnen und Bewohnern des Dorfes (darunter die Ortsbürgermeisterin, Gemeinderäte und aktive Vereinsmitglieder) zwischen 21 und 81 Jahren wurden im Sinne der Grounded Theory hypothesengenerierend kodiert. Dabei fiel auf, dass die Dorfapp immer wieder in einem Atemzug mit den geschlossenen Dorfkneipen genannt wurde. Dorfapp und Dorfkneipe wurden in den Interviews als Kommunikationsräume in einen direkten Zusammenhang gestellt. Diese Beobachtung führt zu der hier behandelten Frage: Inwiefern kann eine Dorfapp als Ersatz für die Dorfkneipe dienen? Dazu werden im Folgenden die unterschiedlichen Funktionen von Kneipen systematisiert, das Dorf Dreis-Brück und die Dorfapp vorgestellt, um dann ihre Funktionen mit jenen der inzwischen oftmals aufgegebenen Kneipen zu vergleichen.

Oft werden nur solche Orte als Kneipe bezeichnet, in denen „,zu primär kommerziellen Zwecken Alkohol ausgeschenkt wird“ (Reinhardt 2006, S. 107). Restaurants und Cafés sind in wissenschaftlichen Arbeiten meist ausgeschlossen (Dröge und Krämer-Badoni 1987; Reinhardt 2006). Hier wird der Begriff ,Dorfkneipe ' in einem weiten Sinne verwendet, wie er im betrachteten Dreis-Brück, aber auch in anderen Dörfern gebraucht wird: Damit ist eine gastronomische Einrichtung gemeint, wo man sich zwar ,zum Bier' trifft, die aber mitunter auch Essen anbietet, wo Familien- oder Firmenfeiern stattfinden und manchmal eine Zimmervermietung dazugehört. In Bayern ist dafür der Begriff (Dorf-)Wirtshaus gebräuchlich (Hopfinger et al. 2013).

In ihrer Untersuchung bayerischer Wirtshäuser bezeichnen Hopfinger et al. das Dorfwirtshaus als „Institution, die positiven Einfluss auf den Gemeinschaftssinn und die Kommunikation der Bürger im Ort ausübt" (Hopfinger et al. 2013, S. 14). Eine ähnliche Bedeutung für den sozialen Zu- sammenhalt im Dorf zeigen auch Arbeiten zu ,village pubs ‘ im ländlichen England (Canduela et al. 2012; Mount und Cabras 2015). Der Kneipe werden dafür mehrere Funktionen zugeschrieben (vgl. Dröge und Krämer-Badoni 1987; Reinhardt 2006; Hopfinger et al. 2013). Sie gelten als:

1. Informations- und Kommunikationsort, wo ein Austausch von Informationen und Neuigkeiten stattfindet;

2. Treffpunkt, an dem Sozialbeziehungen entstehen und gepflegt werden, mit anderen Menschen und dem Wirt über allgemeine und persönliche Probleme gesprochen wird;

3. Ort der Entspannung, beispielsweise durch Spielen, Wetten, Musikhören, Gespräche etc.;

4. Ankunftsort für neue Bewohnerinnen und Bewohner oder Gäste;

5. Emotions- und Erinnerungsraum mit Kontrollfunktion, im Zusammenhang mit Alkoholkonsum, aber auch als Speicher des kulturellen Gedächtnisses.

\section{Dreis-Brück, seine Kneipen und seine Dorfapp}

Wie in vielen ländlichen Regionen gab es auch in den Dörfern der Vulkaneifel oft mehrere Kneipen. „Manche Orte hatten früher vier, fünf Kneipen, heute gibt's vielleicht noch eine, höchstens eine“, erzählt ein Mitarbeiter der Verbandsgemeinde Daun, zu der Dreis-Brück gehört. Seit der Zusammenlegung der Dörfer Dreis und Brück 1974 hielt sich die Einwohnerzahl mit aktuell 815 Personen weitestgehend stabil. Insgesamt gibt es 422 Wohnungen mit einer durchschnittlichen Wohnfläche von $119,8 \mathrm{~m}^{2}$ (Statistisches Landesamt Rheinland-Pfalz 2019) und kaum Leerstand. In den letzten 25 Jahren sind zahlreiche Einrichtungen und Angebote im Dorf geschlossen worden. Einkaufsmöglichkeiten bestehen vor Ort nicht mehr. Das letzte Lebensmittelgeschäft schloss im Jahr 2000, die Sparkasse 2016. Immer wieder betonten die interviewten Personen aber vor allem, dass es in Dreis-Brück früher viele Kneipen gegeben hätte. Dieses ,Früher" ist für viele Bewohnerinnen und Bewohner noch Teil ihrer persönlichen Erinnerungen. Die Dorfchronik beschreibt 1997 vier Gasthäuser für den Ortsteil Dreis und eines in Brück. Die große Bedeutung dieser zeigt sich an dem Hinweis, dass „Brück und sein Gasthaus, das einzige des Ortes, [...] nicht voneinander zu trennen [sind]" (Ortsgemeinde Dreis-Brück 1997, S. 288). Von diesen fünf Gaststätten existiert im Jahr 2020 nur noch ein Gasthaus mit Beherbergung, welches zu Jahresanfang 2020 nach einem Betreiberwechsel geschlossen und ausgerechnet im März, fast zeitgleich mit dem Beginn der sogenannten CoronaKrise wiedereröffnet wurde. Ferner gibt es in der Dreiser Burg am Wochenende ein Café, welches aber eher als Ausflugsziel beschrieben wird und nicht als dörflicher Treffpunkt dient. (Abb. 1 und 2). 


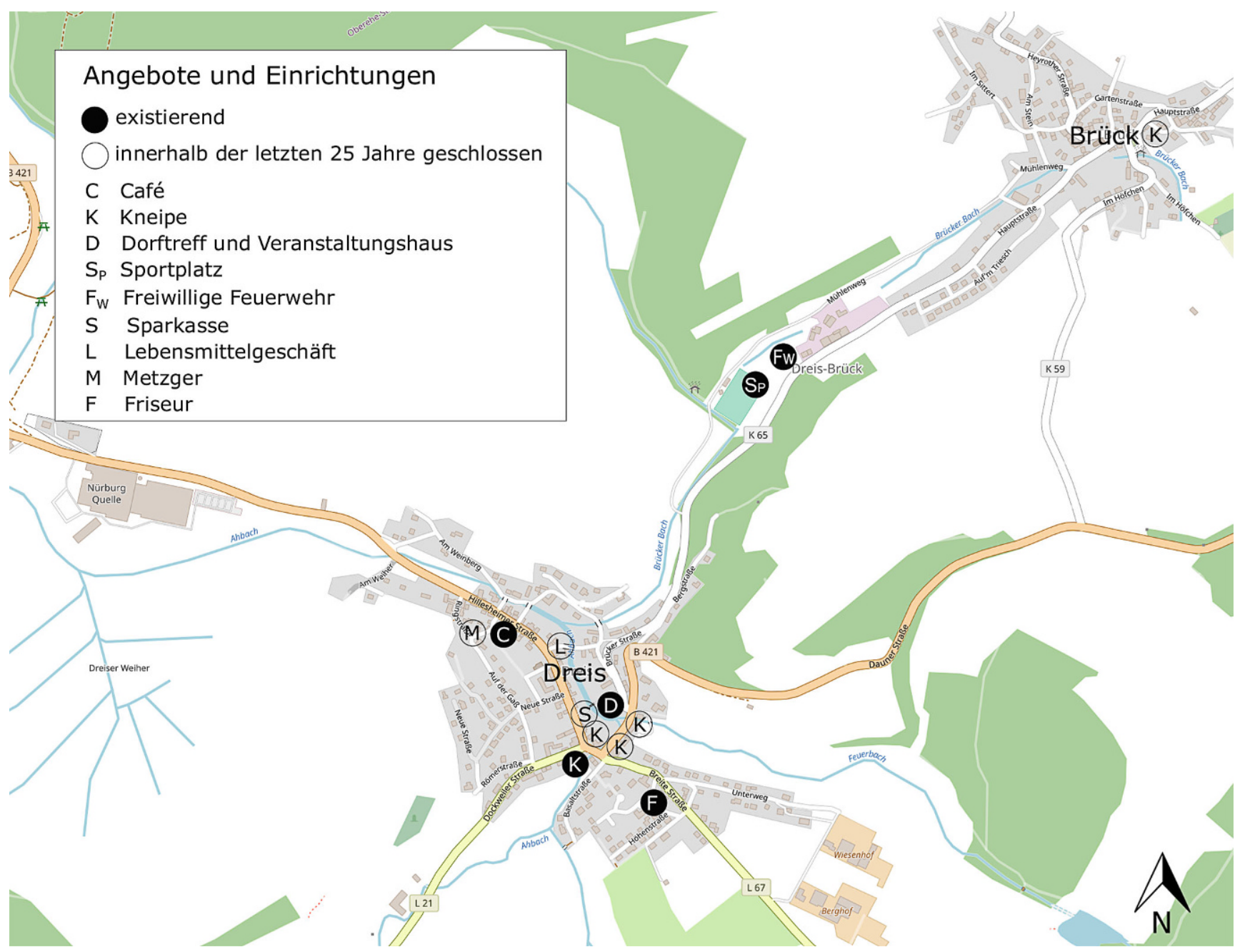

Abb. 1 Dreis-Brück mit seinen Angeboten und Einrichtungen, eigene Darstellung basierend auf OpenStreetMap

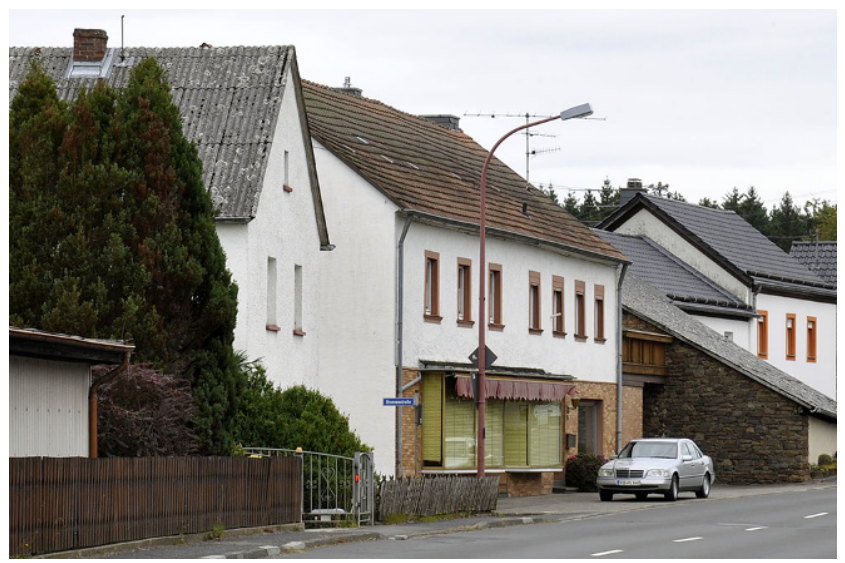

Abb. 2 Ehemaliges Lebensmittelgeschäft in Dreis-Brück, eigenes Foto
Auf Initiative des Verbandsgemeindebürgermeisters fand im April 2017 in Dreis-Brück eine zweitägige Zukunftskonferenz statt, um Probleme und Chancen zu erkennen und Maßnahmen für die Zukunft des Dorfes zu entwickeln. Die etwa 100 teilnehmenden Bewohnerinnen und Bewohner arbeiteten dabei unter anderem fehlende Treffpunkte und mangelhafte Kommunikation im Dorf als Probleme heraus. Die daraufhin gegründete Arbeitsgruppe Коттиnikation wollte dies verbessern: „Wie kann man alternativ zu Treffpunkten dazu kommen, dass Leute sich wieder austauschen? Und da wir uns ja jetzt mittlerweile hier im digitalen Zeitalter befinden, war meine Idee direkt, da müssen wir irgendwas Digitales auf die Beine stellen“, erzählt ein Mitglied der Gruppe.

Nach Internetrecherchen $\mathrm{zu}$ digitalen Tools nahm die Gruppe Kontakt zum Fraunhofer-Institut für Experimentelles Software Engineering IESE auf. Das Institut entwickelte seinerzeit die App Dorffunk im Rahmen des Projektes Digitale Dörfer, welches seit 2015 zunächst in Kooperation mit drei Modellkommunen als Pilotprojekt fungierte (vgl. 
Fraunhofer IESE 2020). Die Dreis-Brücker Arbeitsgruppe stieß mit ihrem Wunsch, ebenfalls diese App nutzen zu können, auf offene Ohren. Ende 2018 stand die leicht angepasste Dorfapp Dreis-Brück zur Verfügung und wurde von der Arbeitsgruppe aktiv über das örtliche Mitteilungsblatt sowie die direkte Ansprache aller Vereine und Gruppen beworben. Die Funktionen der App umfassen den sogenannten ,Plausch“ für Allgemeines und Fragen sowie ,Suche“ und ,Biete‘, wo die Nutzerinnen und Nutzer kurze Einträge schreiben, kommentieren und ,liken“ können. Außerdem können Gruppen für gezielten Austausch angelegt und private Nachrichten geschrieben werden. Längere öffentliche Einträge der als Redakteure Freigeschalteten finden sich unter ,News ' und werden auf eine dazugehörige Webseite verlinkt. Standardmäßig sind Benachrichtigungen über neue Einträge freigegeben, können aber abgeschaltet werden.

Im September 2019 waren 385 Nutzerinnen und Nutzer angemeldet, im Februar 2020 schon 450, von denen 119 mindestens einmal aktiv etwas gepostet oder kommentiert hatten. Zehn Nutzerinnen und Nutzer posten regelmäßig Informationen. Dies sind überwiegend Engagierte des Dorfes, ihre Mitteilungen betreffen insbesondere die Spielergebnisse der örtlichen Sportvereine, Termine im Dorftreff oder der Wandergruppe, Informationen der Ortsbürgermeisterin, des Gemeinderates oder der freiwilligen Feuerwehr. Darüber hinaus werden Mitfahrgelegenheiten geboten und gesucht, Gegenstände verschenkt oder verkauft und während der sogenannten Corona-Krise, immer wieder Fensterkonzerte organisiert. Auch Warnungen, z. B. vor einem umgestürzten Baum oder vor einer Gruppe Trickbetrüger, oder Fragen, z.B. nach der Startzeit des Karnevalsumzugs, werden gepostet. Da die Nutzung der App anonym erfolgt und nur der Vorname mit abgekürztem Nachnamen sichtbar ist, ist auch die AG Kommunikation unsicher, welche Personen tatsächlich erreicht werden, aber, so einer der Engagierten, ,es ist gefühlt schon gemischt. Hauptsächlich natürlich das mittlere Alter ... eher ältere als ganz junge." Ein anderer Vertreter berichtet hingegen: „Die Jugend setzt zu 100\% darauf. [...] wenn es über das Lebensalter von 70 hinausgeht, dann wird es wieder dünner." Insgesamt geht die Arbeitsgruppe davon aus, dass die Hälfte der Dorfbewohnerinnen und -bewohner die App mindestens passiv (nur lesend) nutzt und hofft, dass ,einer in der Familie die App hat“.

\section{Dorfapp und Kneipe in ihren Funktionen}

Informations- und Kommunikationsort: Die Funktion von Gaststätten ,als Orte, an denen private und öffentliche Nachrichten verbreitet werden [...], nimmt allerdings ab, da andere Möglichkeiten zur Informationsübermittlung (u. a. Internet, Social Media) entstanden sind“ (Franz 2020, S. 96). In Dreis-Brück scheint es genau diese Funktion zu sein, die besonders vermisst wird und die zur oben erwähnten Arbeitsgruppe Kommunikation führte. So bezeichnet ein Bewohner die weggefallenen Kneipen als „die absolute Informationsbörse“. Ein anderer bemängelt die Kommunikation im Dorf, ,weil, diese Kommunikationsorte, die wir bis dato hatten, Kneipen, Restaurants, die sind ja immer weiter weggebrochen [...] Da ist jetzt auch die Dorfapp so ein bisschen in die Bresche gesprungen und hat die Lücke auch so ein bisschen aufgefüllt." Als digitaler Kommunikationsraum soll die App ,eine einfache Möglichkeit [bieten], zwanglos irgendwo Informationen auszutauschen“, betont einer der Engagierten.

Die Dorfapp befördert so die „Kommunikation als einen (zweckrationalen) Austausch von Informationen“ (Fiehler 1990, S. 107) und erfüllt somit teilweise die von den Bewohnerinnen und Bewohnern gewünschte Funktion des Kommunikationsraumes. Jüngere Vorstellungen zur kommunikativen Konstruktion von Räumen (Christmann 2016) aber verstehen Kommunikation immer auch als kommunikatives Handeln, also aufeinander bezogenes Handeln mittels unterschiedlicher Zeichen (Keller et al. 2013). Dass ein solches Verständnis von Kommunikation nicht nur theoretisch ist, zeigt beispielhaft die Aussage einer aktiven Nutzerin: ,[Die App] wird nach wie vor ja überwiegend eher als Informationsplattform benutzt, für Bekanntmachungen [...] und weniger in der Interaktion, also in der Kommunikation miteinander. [...] manches oder vieles bleibt halt auch so unkommentiert". Der Wunsch nach mehr Austausch über die App deckt sich mit der Einschätzung eines Engagierten, wonach bisher nur 68 Nutzerinnen und Nutzer einen Eintrag kommentiert hätten. Er wünscht sich, ,dass da noch ein regerer Austausch stattfinden könnte".

Treffpunkt: In der Kneipe „hat man Karten miteinander gespielt, da hat man mal einen über den Durst getrunken. Das wurden ja ganz tolle Erlebnisse [...] und diese Kultur kriegst du natürlich auf die Schnelle mit so einem digitalen Medium nicht mehr aufgebaut", ist sich einer der Engagierten sicher. Physische Treffpunkte mit ihrem Geruch, ihrer Atmosphäre und Lautstärke, aber auch in der Unverbindlichkeit und Zufälligkeit sozialer Beziehungen kann die Dorfapp am wenigsten ersetzen. Es fehlen der „Raum der Kneipenöffentlichkeit" (Dröge und Krämer-Badoni 1987, S. 209) und die ,hohe sozialisierende Kraft des Conviviums“ (Matthiesen 2005, S. 10), welche durch gemeinsames Essen und Trinken entsteht. Auch mit Dorfapp braucht es Treffpunkte, um digitale und analoge Kommunikation zusammenzuführen.

Ort der Entspannung: Geht es um die Kneipe als Ort der Entspannung, wird oft auf gemeinsames Spielen verwiesen, wie auch im obigen Zitat. Dies ist theoretisch digital möglich, wenn auch nicht über die Dorfapp. Interessanter ist der Hinweis auf eine besondere Form der Entspannung: schwei- 
gend am Tresen ein Bier trinken, lesen, nachdenken oder einfach nur das Geschehen beobachten (Dröge und KrämerBadoni 1987, S. 208). Hier zeigt sich eine klare Fehlstelle der Dorfapp und anderer digitaler Kommunikationsmittel: Gehören Kneipengäste auch ohne aktives Einmischen in Gespräche oder Spiele dazu, so geschieht das schweigende Mitlesen - vielleicht sogar zur Entspannung mit einem Bier auf dem Sofa - der Dorfapp unter Ausschluss der Öffentlichkeit.

Ankunftsort: Durch ihre Wirkung in den öffentlichen Raum werden Kneipen zu Ankunftsorten für Fremde. Ist die Dorfapp auch Fremden bekannt und zugänglich, kann sie einen digitalen Ankunftspunkt bieten, wie die Autorin erleben durfte. Vor dem zweiten Forschungsaufenthalt in Dreis-Brück war in der Dorfapp zu lesen, dass am Tag der Ankunft eine Feier im Dorftreff stattfand. So ergab sich die Möglichkeit, informell das Forschungsprojekt vorzustellen und in den Alltag der Dorfgemeinschaft einzutauchen. Erneut zeigt sich die Bedeutung der Verknüpfung digitaler Räume und analoger Orte: Der digitale Raum kann dazu führen, dass ein persönliches Kennenlernen am analogen Ort überhaupt stattfindet. Oder wie es ein Dorfbewohner beschreibt: „Also dieses Medium soll nicht den Kontakt an sich auf die digitale Ebene bringen, sondern soll einfach nur mal ein Anstoß sein, dass man normal miteinander redet."

Emotions- und Erinnerungsraum mit Kontrollfunktion: Die Funktion der Kneipe ,als Bündelung emotionaler und edukativer Aspekte“ (Hopfinger et al. 2013, S. 14), kann die Dorfapp ebenfalls nicht ersetzen. Eine Kontrollfunktion besteht einzig hinsichtlich der verbalen Äußerungen durch das mögliche Eingreifen von Moderatorinnen und Moderatoren in die Posts der App. Dass dies im ersten Jahr nur einmal notwendig war, zeigt jedoch, dass die Hemmschwelle innerhalb der Dorfapp recht hoch sein dürfte. Im Gegensatz zur Kneipe spielt Alkoholkonsum selbstredend keine unmittelbare Rolle und auch als Emotionsraum fungiert die Dorfapp nicht. Wird die Kneipe jedoch weniger als Affektnische und mehr als Teil des kulturellen Gedächtnisses verstanden (vgl. Reinhardt 2006), ist bisher noch offen, ob sich nicht über die Dorfapp eine Art kommunikatives Gedächtnis (Knoblauch 1999) des Dorfes etablieren kann. Über die in der App geposteten Informationen wird durchaus geredet, man frage sich untereinander: „Hast du gelesen, da und da?" Mitunter kann die Dorfapp über die Zeit zu einem Speicher der Geschichte des Dorfes werden und damit das kommunikative Gedächtnis befördern.

\section{Fazit}

Die obigen Ausführungen verdeutlichen, dass eine App nicht vollständig eine Kneipe ersetzen kann. Ein detaillierter Blick auf die der Kneipe zugeschriebenen Funktionen zeigt aber, dass die Dorfapp erstaunlich viele Funktionen zumindest teilweise ersetzen kann: Sie kann bis zu einem gewissen Grad zum digitalen Kommunikationsraum werden, in Ansätzen auch einen Ankunftsraum bieten und langfristig mitunter das kommunikative Dorfgedächtnis befördern. Die im Dorf aber so bedeutsame Funktion der Dorfkneipe als Treffpunkt mit ihren Möglichkeiten kommunikativen Handelns und ihrer Wirkung in den öffentlichen Raum kann die Dorfapp nicht ersetzen. Ein Dorfbewohner bringt es auf den Punkt: „Das ist ein kleiner Ersatz [...] Aber so eine Kneipe an sich, was die alles darüber hinaus noch bietet, kann so ein digitales Medium nie ersetzen."

Bei allen Hoffnungen, die auf der Digitalisierung liegen, gilt es zukünftig digitale Räume und analoge Orte bewusst miteinander zu verknüpfen. Eine systematische Betrachtung der Probleme, die mithilfe digitaler Tools gelöst werden sollen, sowie der analogen Raumeigenschaften, kann helfen, solche Verknüpfungen herzustellen.

Funding Open Access funding enabled and organized by Projekt DEAL.

Open Access Dieser Artikel wird unter der Creative Commons Namensnennung 4.0 International Lizenz veröffentlicht, welche die Nutzung, Vervielfältigung, Bearbeitung, Verbreitung und Wiedergabe in jeglichem Medium und Format erlaubt, sofern Sie den/die ursprünglichen Autor(en) und die Quelle ordnungsgemäß nennen, einen Link zur Creative Commons Lizenz beifügen und angeben, ob Änderungen vorgenommen wurden.

Die in diesem Artikel enthaltenen Bilder und sonstiges Drittmaterial unterliegen ebenfalls der genannten Creative Commons Lizenz, sofern sich aus der Abbildungslegende nichts anderes ergibt. Sofern das betreffende Material nicht unter der genannten Creative Commons Lizenz steht und die betreffende Handlung nicht nach gesetzlichen Vorschriften erlaubt ist, ist für die oben aufgeführten Weiterverwendungen des Materials die Einwilligung des jeweiligen Rechteinhabers einzuholen.

Weitere Details zur Lizenz entnehmen Sie bitte der Lizenzinformation auf http://creativecommons.org/licenses/by/4.0/deed.de.

\section{Literatur}

Bade D, Nehls A (2018) Warum immer mehr Kneipen in Brandenburg schließen. Märkische Allgemeine Zeitung

Canduela J, Cabras I, Raeside R (2012) The relation of village and rural pubs with community life and peoples well-being in Great Britain. Ger J Agric Econ 61:265-274

Christmann G (Hrsg) (2016) Zur kommunikativen Konstruktion von Räumen. Springer, Wiesbaden

Dröge F, Krämer-Badoni T (1987) Die Kneipe; Zur Soziologie einer Kulturform oder „2 Halbe auf mich!“. Suhrkamp, Frankfurt am Main

Fiehler R (1990) Kommunikation, Information und Sprache; Alltagsweltliche und wissenschaftliche Konzeptualisierungen und der Kampf um die Begriffe. In: Weingarten R (Hrsg) Information ohne Kommunikation? Die Loslösung der Sprache vom Sprecher. Fischer, Frankfurt a.M., S 99-128

Franz M (2019) Gaststätten als Teil der lokalen Ökonomie. In: Henn S, Behling M, Schäfer S (Hrsg) Lokale Ökonomie - Konzepte, Quartierskontexte und Interventionen. Springer, Berlin, Heidelberg, S 1-19 
Franz M (2020) Treffpunkt Gaststätte - warum sich Kommunen stärker um ihre Kneipen und Restaurants kümmern sollten. Standort 44:93-98

Fraunhofer IESE (2020) DorfFunk - Digitale Dörfer. https://www. digitale-doerfer.de. Zugegriffen: 4. Apr. 2020

Gyr U (1997) Kneipen als städtische Soziotope; Zur Bedeutung und Erforschung von Kneipenkulturen. Osterr Z Volkskd 94: 97-116

Henkel G (2018) Rettet das Dorf! Was jetzt zu tun ist. dtv, München

Hopfinger H, Kohnle F, Wätzold T (2013) Genuss mit Geschichte? Die Wirtshauskultur in Bayern im Wandel. Kath. Universität Eichstätt-Ingolstadt, Eichstätt

Keller R, Reichertz J, Knoblauch H (Hrsg) (2013) Kommunikativer Konstruktivismus; Theoretische und empirische Arbeiten zu einem neuen wissenssoziologischen Ansatz. Springer VS, Wiesbaden

Knoblauch H (1999) Das kommunikative Gedächtnis. In: Honegger C, Hradil S, Traxler F (Hrsg) Grenzenlose Gesellschaft? VS, Wiesbaden, $\mathrm{S} 733-748$

Matthiesen U (2005) Kulinarik und Regionale Entwicklung unter besonderer Berücksichtigung von „Mark und Metropole“ (Antrittsvorlesung am 27. Mai 2003, Berlin)
Mount M, Cabras I (2015) Community cohesion and village pubs in Northern England; an econometric study. Reg Stud 50:1203-1216. https://doi.org/10.1080/00343404.2014.989150

Norddeutscher Rundfunk (2020) Wie Dörfer ihre Kneipen retten. https://www.ndr.de/fernsehen/sendungen/45_min/Wie-Doerferihre-Kneipen-retten, sendung913052.html. Zugegriffen: 27. März 2020

Ortsgemeinde Dreis-Brück (1997) Dreis-Brück; Eine Dorfchronik

Pätzold R (2012) Tischlein, deck dich! Über städtische Gastronomielandschaften und was Stadtplanung damit zu tun haben könnte. In: Schrenk M, Popovich V, Zeile P, Elisei P (Hrsg) REAL CORP 2012-re-mixing the city. CORP, Schwechat-Rannersdorf, S 911-921

Reinhardt JD (2006) Die Kneipe als Generator emotionaler Erinnerungen. Psychol Gesellschaftskrit 30:105-129

Sixtus F, Slupina M, Sütterlin S, Amberger J, Klingholz R (2019) Teilhabeatlas Deutschland. Berlin-Institut f. Bevölkerung u. Entwicklung, Berlin

Statistisches Landesamt Rheinland-Pfalz (2019) Mein Dorf, meine Stadt. Dreis-Brück. http://www.infothek.statistik.rlp.de/Meine Heimat/content.aspx $? \mathrm{id}=103 \& \mathrm{~g}=0723301020 \& \mathrm{l}=3 \& \mathrm{tp}=8191$. Zugegriffen: 30. Juli 2020 